(C) 2020. This manuscript version is made available under the CCBY-NC-ND 4.0 license http:/creativecommons.org/licenses/by-nc$\mathrm{nd} / 4.0 /$

\title{
Early Growth Patterns and Cardiac Structure and Function at Midlife: Northern Finland 1966 Birth Cohort Study
}

\author{
Nelli Korpela ${ }^{1}$, Kari Kaikkonen ${ }^{1}$, Juha Auvinen ${ }^{2,3}$, Mikko P. Tulppo $^{1}$, Juhani \\ Junttila $^{1}$, Juha Perkiömäki ${ }^{1}$, Marjo-Riitta Järvelin ${ }^{2,3,4,5,6}$, Heikki V. Huikuri ${ }^{1}$, \\ Antti M. Kiviniemi ${ }^{1}$ \\ ${ }^{1}$ Research Unit of Internal Medicine, Medical Research Center Oulu, Oulu University \\ Hospital and University of Oulu, Oulu, Finland \\ ${ }^{2}$ Center for Life Course Health Research, Faculty of Medicine, University of Oulu, Oulu, \\ Finland \\ ${ }^{3}$ Unit of Primary Health Care, Medical Research Center Oulu, Oulu University Hospital, \\ Oulu, Finland \\ ${ }^{4}$ Biocenter Oulu, University of Oulu, Oulu, Finland \\ ${ }^{5}$ Department of Epidemiology and Biostatistics, MRC-PHE Centre for Environment \& \\ Health, School of Public Health, Imperial College London, London, UK \\ ${ }^{6}$ Department of Life Sciences, College of Health and Life Sciences, Brunel University \\ London, UK
}

Supported by the Academy of Finland (no. 285547), Finnish Foundation for Cardiovascular Research, European Union's Horizon 2020 research and innovation program (633595, EU H2020-PHC-2014 633595, DynaHEALTH, EU H2020-SC1-2016-2017, LIFECYCLE, EU H2020-HCO-2014, iHEALTH), Yrjö Jahnsson Foundation, Aarne Koskelo Foundation, and Oulu University Scholarship Foundation. NFBC1966 received financial support from University of Oulu (no. 24000692), Oulu University Hospital (no. 24301140), and ERDF European Regional Development Fund (no. 539/2010 A31592). Sponsors had no role in study design, the collection, analysis or interpretation of the data, writing of the report or the decision to submit the manuscript for publication. The authors declare no conflicts of interest. Portions of this study were presented at the European Society of Cardiology Congress, August 31-September 4, 2019, Paris, France. 


\section{Abstract}

Objectives: To evaluate the influence of early growth patterns, that have previously been associated with later cardiometabolic risk, on cardiac left ventricular (LV) structure and function in midlife.

Study design: A subpopulation of the Northern Finland Birth Cohort 1966 took part in follow-up, including echocardiography $(n=1155)$ at the age of 46 years. Body mass index (BMI) growth curves were modeled based on frequent anthropometric measurements in childhood. Age and BMI at adiposity peak (AP, $\mathrm{n}=482$, mean age 9.0 months) and at adiposity rebound ( $\mathrm{AR}, \mathrm{n}=586$, mean age 5.8 years) were determined. Results are reported as unstandardized beta $(\beta)$ or odds ratio (OR) with $95 \%$ CIs for $1 \mathrm{SD}$ increase in early growth variable.

Results: Earlier adiposity rebound was associated with increased LV mass index $\left(\beta=-4.10 \mathrm{~g} / \mathrm{m}^{2}[-6.9,-1.3] ; \mathrm{P}=.004\right)$ and $\mathrm{LV}$ end-diastolic volume index $(\beta=-2.36$ $\left.\mathrm{mL} / \mathrm{m}^{2}[-3.9,-0.84] ; \mathrm{P}=.002\right)$ as well as with eccentric LV hypertrophy $(\mathrm{OR}=0.54$ $[0.38,0.77] ; \mathrm{P}=.001)$ in adulthood in males. $\mathrm{BMI}$ at adiposity rebound was directly associated with $\mathrm{LV}$ mass index $\left(\beta=2.33 \mathrm{~g} / \mathrm{m}^{2}[0.80,3.9] ; \mathrm{P}=.003\right)$. Higher BMI at both adiposity peak and at adiposity rebound were associated with greater LV enddiastolic volume index $\left(\beta=1.47 \mathrm{~mL} / \mathrm{m}^{2} ;[0.51,2.4], \beta=1.28 \mathrm{ml} / \mathrm{m}^{2}[0.41,2.2]\right.$, respectively) and also with eccentric $\mathrm{LV}$ hypertrophy $(\mathrm{OR}=1.41[1.10,1.82]$, $\mathrm{OR}=1.53[1.23,1.91]$, respectively) and $\mathrm{LV}$ concentric remodeling $(\mathrm{OR}=1.38$ [1.02, 1.87], $\mathrm{OR}=1.40[1.06,1.83]$, respectively) in adulthood $(\mathrm{P}<.05$ for all). These relationships were only partly mediated by adult BMI.

Conclusions: Early growth patterns in infancy and childhood contribute to cardiac structure at midlife.

\section{Keywords}

left ventricular structure; left ventricular mass; left ventricular hypertrophy; childhood growth; body mass index; adiposity rebound

\section{Abbreviations}

BMI, Body mass index; E/e', Ratio of early diastolic mitral inflow velocity and mitral septal early diastolic velocity; LA, Left atrial; LV, Left ventricular; LVEF, Left ventricular ejection fraction; LVM, Left ventricular mass; NFBC, Northern Finland Birth Cohort; RWT, Relative wall thickness; SES, Socioeconomic status 
Increased left ventricular (LV) mass (LVM) is independently related to cardiovascular morbidity and mortality in adulthood (1-3). Prenatal and childhood growth patterns have been related to adult cardiovascular risk (4-7), but the information on the long-term effects of early growth to later cardiac structure is limited. It has been suggested that individuals born pre-term have increased LVM and changes in cardiac function in early adulthood (8). The few previous studies investigating the relationship between birth weight and adult LV structure have found variable results but generally no evidence of an association (9-13).

Obesity and elevated blood pressure are major factors leading to excessive cardiac growth, beyond that of normal somatic growth, in children (14-16). Adaptations in cardiac structure have been observed in children with obesity from as early as 2 years of age (16). LVM has been shown to track throughout childhood growth and into later life $(17,18)$. Increased body mass index (BMI) beginning in childhood has been associated with increased LVM and altered LV geometric patterns in adulthood $(9,19-22)$.

Infant and childhood BMI growth trajectory data have shown their value as a tool to estimate obesity and cardiometabolic risk in later life (5). After birth, the BMI-for-age growth curve typically rises until the adiposity peak of infancy (before the age of 1 year) and then decreases before increasing again in later childhood. The nadir of BMI is often referred to as adiposity rebound (23). Early timing of adiposity rebound has been associated with increased risk of obesity and a disadvantageous cardiometabolic profile in later life and has been suggested to be a critical phase in childhood growth development $(5,6,23)$. Later timing of and a higher BMI at adiposity peak has been suggested to associate with later overweight $(6,24)$.

The aim of the present study was to assess the associations of birth characteristics and infant and childhood growth patterns with cardiac structure and function in midlife (46 years of age) in the Northern Finland Birth Cohort (NFBC) 1966. We hypothesized that birth characteristics, earlier timing of adiposity rebound, as well as a higher BMI at adiposity peak and at adiposity rebound would associate with modifications in adult cardiac structure. 


\section{Methods}

The study population is a sample of the large prospective NFBC 1966, which covers $96.3 \%$ of all births in 1966 in the 2 northernmost provinces of Finland $(\mathrm{n}=$ 12058 live births) (25). Their mothers' recruitment started from the $16^{\text {th }}$ gestational week, and extensive data have been collected on the participants' growth, health, and lifestyle until midlife. The study has been conducted according to the Declaration of Helsinki and approved by the Ethical Committee of the Northern Ostrobothnia Hospital District. All participants have provided their written informed consent.

Maternal data were collected by questionnaire during the $24^{\text {th }}-28^{\text {th }}$ gestational weeks. Based on these questionnaire data, family socioeconomic status (SES), and maternal pre-pregnancy BMI were determined. Family SES was based on paternal occupation at recruitment (maternal occupation, if mother was single). SES categories adapted from Järvelin et al were merged into the 4 most relevant categories (high, middle, low, farmer) (25). Birth weight $( \pm 5 \mathrm{~g})$ was measured immediately after delivery. Gestational age was determined from the mother's last menstrual period.

BMI at various ages was calculated from frequent anthropometric measurements during infancy and childhood collected from child welfare clinical records. The growth modeling methods used in this study have been described in detail elsewhere (6). Polynomial mixed effect models did not satisfactorily fit to the data from birth to adolescence ( $0-13$ years of age) because of the complex shape of the typical BMI growth curve, and consequently the modeling of growth in infancy $(0-1.5$ years, $n=3265)$ and childhood $(>1.5-13$ years, $n=4121)$ was carried out separately. Growth variables (age and BMI at adiposity peak and at adiposity rebound) were determined from cubic models in age, with random effects for the intercept (baseline BMI) and slope term. Models were adjusted for sex, and sex interactions were taken into account. Age at adiposity peak was determined as the age at maximum BMI between 0.25 and 1.25 years, and age at adiposity rebound as the age at minimum BMI between 2 and 8.5 years. The cut-off points were chosen based on descriptive analysis of growth curves. Individuals with fewer than 3 measurements in each period were excluded. Approximately 6 measurements in infancy (from 2 weeks to 1.5 years) and 10 measurements in childhood (from 1.5 years to 13 years) per participant were available.

Postal questionnaires on the participant's health status and lifestyle were conducted when the participants were age 14,31 , and 46 years. The response rates 
were $97 \%$ at 14 years $(\mathrm{n}=11399), 75 \%$ at 31 years $(\mathrm{n}=8767)$, and $66 \%$ at 46 years $(\mathrm{n}=6825)$. Physical activity at 14,31 , and 46 years of age was assessed based on questionnaire answers as previously described (26). Latent class analysis was used to acquire clusters in which the individuals had a similar profile of physical activity from adolescence to midlife and 3 lifelong physical activity trajectory groups (active, semi-active, and inactive) were formed $(26,27)$. At the age of 46 years, a comprehensive follow-up of the cohort participants was carried out in the years 2012-2014. Participants were invited to clinical examinations at the Center for Life Course Health Research (University of Oulu) with 3 laboratory units (Oulu, Southern, and Northern Finland); a total of 5861 (57\%) participated. Clinical examination included anthropometric measurements, blood sampling, and cardiovascular health status. Venous blood samples were drawn for the analysis of glycemic status. On a separate day after 12 hours of fasting, a 2-hour oral glucose tolerance test was conducted for participants without medication for diabetes. Diabetes diagnosis was made according to the criteria issued by the World Health Organization (fasting plasma glucose $\geqslant 7.0 \mathrm{mmol} / \mathrm{L}$ or 2 -hour glucose $\geqslant 11.1$ $\mathrm{mmol} / \mathrm{L}$ in oral glucose test or glycated hemoglobin $\geqslant 6.5 \%)(28)$. Systolic and diastolic blood pressure were measured in a seated position in the right arm after 15 minutes of rest (the 2 out of 3 lowest systolic values and the corresponding diastolic values were averaged) with an automated sphygmomanometer (Omron M10; Omron Healthcare, Kyoto, Japan).

A randomly selected subpopulation $(\mathrm{n}=1155)$ was enrolled for echocardiographic examinations (Oulu laboratory unit only). Comprehensive transthoracic 2-dimensional echocardiography was performed on-line by an experienced cardiologist, using the General Electric Vivid E9 device with a M5SD 1.5/4.6 MHz sector transducer for cardiovascular imaging (GE Health Medical, Horten, Norway). All measurements were performed according to the American Society of Echocardiography guidelines (29). We focused on the following echocardiographic measurements as outcomes: LVM, LV end-diastolic volume, interventricular septal thickness, posterior wall thickness, and left atrial (LA) endsystolic volume to evaluate LV and LA structure, as well as LV ejection fraction (LVEF) to evaluate systolic and E/e' (ratio of early diastolic mitral inflow velocity and mitral septal early diastolic velocity) diastolic function. In addition, global longitudinal strain, a novel indicator useful in assessing early stage systolic dysfunction, was assessed off-line using the EchoPac 7 software (automated function imaging; GE Health Medical, Horten, Norway) (30,31). Cardiac structural measures were indexed to body surface area to account for body size. Relative wall 
thickness (RWT) was calculated using the formula (posterior wall thickness + interventricular septal thickness)/LV end-diastolic diameter. Four LV geometric remodeling categories were established based on LVM index and RWT: normal geometry (normal LVM index and normal RWT), concentric remodeling (normal LVM index and increased RWT), eccentric hypertrophy (increased LVM index and normal RWT), and concentric hypertrophy (increased LVM index and increased RWT) (Figure 1; available at www.jpeds.com) (29). LV hypertrophy cut-off values based on LVM indexed to body surface area were $>115 \mathrm{~g} / \mathrm{m} 2$ for men and $>95 \mathrm{~g} / \mathrm{m} 2$ for women and cut-off value for increased RWT was $>0.42$ (29).

Participants with suboptimal quality of echocardiographic imaging $(n=87)$ or significant heart defects $(\mathrm{n}=36)$ were excluded. For the analyses including cardiac function, participants with heart rate $>85$ beats/min during echocardiography ( $\mathrm{n}=$ 105) were also excluded. A flow chart of the study population is presented (Figure $2)$.

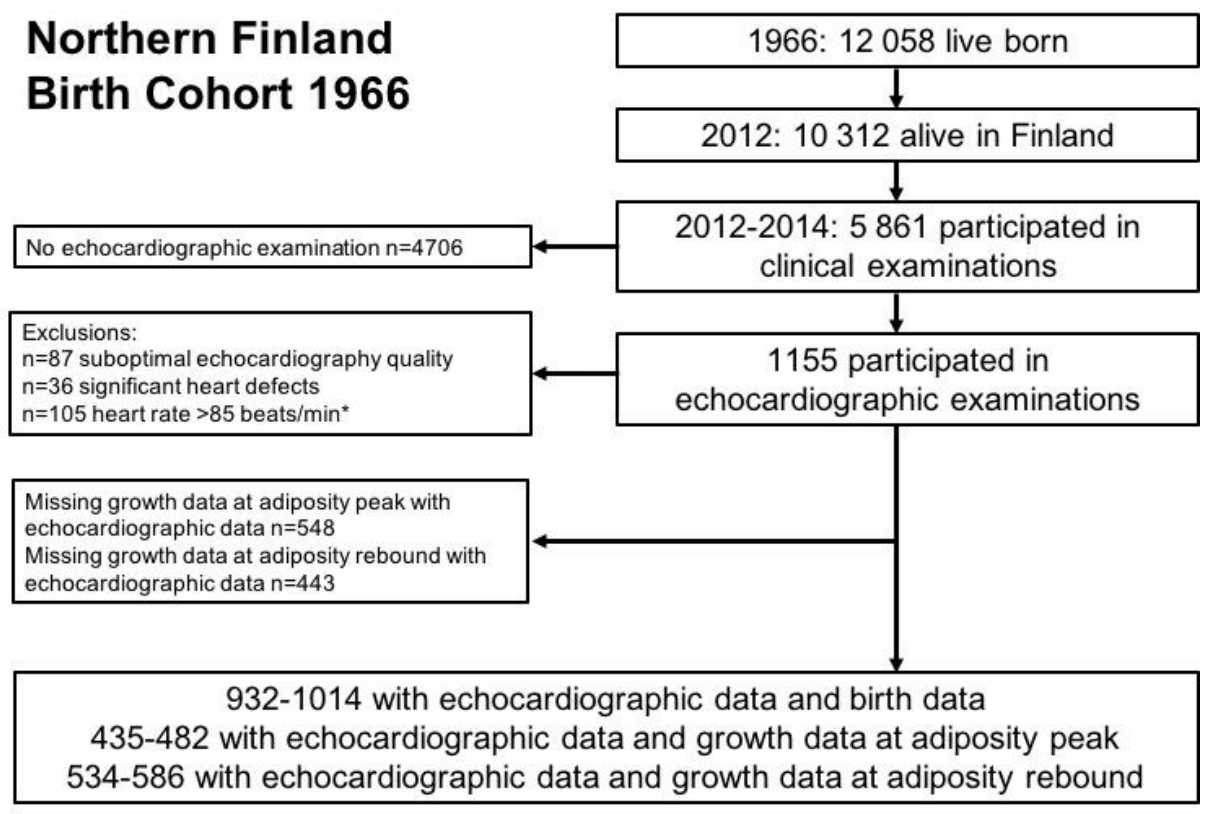

Fig. 2. Flowchart of study population, NFBC 1966. * Participants with heart rate $>85$ beats/minute were excluded only from the analyses including measures of cardiac function. Not all echocardiographic measures were available for each participant which explains the variation in final $\mathbf{n}$. $\mathbf{N}$ for analyses with global strain are not shown in the Figure, because they were significantly smaller than for other echocardiographic 
measures: $n=703$ with birth data, $n=338$ with adiposity peak data, and $n=414$ with adiposity rebound data.

The data were analyzed using SPSS software (IBM SPSS Statistics 24; IBM Corp, Armonk, New York). The distributions of the dependent variables were verified for normality (Gaussian distribution). Sex interactions in the associations between growth variables and echocardiographic measures were tested by ANCOVA for continuous echocardiographic variables and by multinomial logistic regression analysis for categorized LV remodeling classes (sex*growth variable [in tertiles]). When interaction by sex was present, sexes were analyzed separately. Linear regression models were used to assess the relationships between growth variables (gestational age, birth weight, age and BMI at adiposity peak and at adiposity rebound) and adult echocardiographic variables (LVM index, LV end-diastolic volume index, interventricular septal thickness index, posterior wall thickness index, RWT, LA end-systolic volume index, LVEF, global strain, and E/e'). For LV remodeling categories, analyses were conducted with multinomial logistic regression models. Results are reported as unstandardized beta ( $\beta$ ) or OR with $95 \%$ CIs per $1 \mathrm{SD}$ increase in independent variable.

Associations between early growth and cardiac structure and function in adulthood were adjusted first for sex (model 1), then additionally for birth weight, maternal pre-pregnancy BMI, and family SES (and gestational age for analysis with birth weight as main independent variable) (model 2). Subsequently, associations were further adjusted for lifelong physical activity as well as adult cardiovascular risk factors and morbidity (systolic and diastolic blood pressure, heart rate during echocardiographic examination, diabetes based on previous or new diagnosis, cardiac diseases, and antihypertensive medication) (model 3); and further, for adult BMI (and nonindexed values RWT, LVEF, global strain, E/e' were also adjusted for adult height) (model 4). Finally, associations between measures at adiposity rebound and cardiac structure and function in adulthood were additionally adjusted for BMI at adiposity peak. Variables were continuous when applicable. No significant collinearity between the independent variables was present (variance inflation factor $<5$ for all independent variables in the final models). Histograms of regression standardized residual frequency and normal P-P plots of regression standardized residuals were visually inspected to ensure that the models met the analysis assumption. Among the included participants, there were some missing data in dependent and independent variables as well as covariates. We used 
maximum available participant-approach and the variation in the number of participants in specific analyses are noted in the results.

\section{Results}

Table I describes the characteristics of the study population. Some differences by sex were observed. Male participants were born larger and heavier than female participants, and their BMI at adiposity peak was higher than in female participants. At 46 years of age, male participants had a more disadvantageous cardiometabolic profile than female participants. Male participants had larger hearts (LVM and LV end-diastolic volume, interventricular septal thickness, LA end-systolic volume). Female participants had slightly higher LVEF and lower global strain, but a higher E/e'.

Table 1. Characteristics of the study population

\begin{tabular}{|c|c|c|c|}
\hline Characteristics & men & women & $P$ value \\
\hline \multicolumn{4}{|l|}{ Growth variables } \\
\hline Maternal pre-pregnancy BMI $\left(\mathrm{kg} / \mathrm{m}^{2}\right)$ & $23.1(3.0)$ & $23.0(3.3)$ & .794 \\
\hline Gestational age (wk) & $40.0(1.8)$ & $40.2(1.9)$ & .189 \\
\hline Birth weight (g) & $3585(540)$ & $3447(480)$ & $<.001$ \\
\hline Birth length $(\mathrm{cm})$ & $50.7(2.1)$ & $49.9(2.0)$ & $<.001$ \\
\hline Age at adiposity peak $(y)^{\star}$ & $0.75(0.06)$ & $0.75(0.06)$ & .386 \\
\hline Age at adiposity rebound $(y)^{\dagger}$ & $5.8(0.82)$ & $5.7(0.83)$ & .172 \\
\hline BMI at adiposity peak $\left(\mathrm{kg} / \mathrm{m}^{2}\right)^{*}$ & $18.1(1.0)$ & $17.8(1.0)$ & .001 \\
\hline $\mathrm{BMI}$ at adiposity rebound $\left(\mathrm{kg} / \mathrm{m}^{2}\right)^{\dagger}$ & $15.4(1.0)$ & $15.3(1.1)$ & .167 \\
\hline \multicolumn{4}{|l|}{ Lifelong physical activity } \\
\hline & active $100(27 \%)$ & $165(32 \%)$ & \\
\hline & semi-active 188 (51\%) & $215(42 \%)$ & \\
\hline & inactive $83(22 \%)$ & $134(26 \%)$ & .153 \\
\hline \multicolumn{4}{|l|}{ Cardiometabolic outcomes at 46 y } \\
\hline Weight (kg) & $85.2(12)$ & $70.3(12)$ & $<.001$ \\
\hline Height $(\mathrm{cm})$ & $178.7(6.2)$ & $165.2(5.8)$ & $<.001$ \\
\hline $\mathrm{BMI}\left(\mathrm{kg} / \mathrm{m}^{2}\right)$ & $26.7(3.4)$ & $25.8(4.5)$ & $<.001$ \\
\hline Systolic blood pressure (mm Hg) & $129(13)$ & $119(16)$ & $<.001$ \\
\hline
\end{tabular}




\begin{tabular}{|c|c|c|c|}
\hline Diastolic blood pressure (mm Hg) & $85(8.9)$ & $81(11)$ & $<.001$ \\
\hline Diabetes (n) & $15(3.4 \%)$ & $33(5.8 \%)$ & .075 \\
\hline Heart diseases (n) & $8(1.8 \%)$ & $8(1.4 \%)$ & .608 \\
\hline Antihypertensive medication ( $n$ ) & $60(13.5 \%)$ & $65(11.4 \%)$ & .299 \\
\hline \multicolumn{4}{|l|}{ Echocardiographic features at 46 y } \\
\hline Heart rate $(\mathrm{bpm})$ & $66(12)$ & $69(10)$ & $<.001$ \\
\hline $\operatorname{LVM}(g)$ & $210.0(51.5)$ & $148.2(34.9)$ & $<.001$ \\
\hline LVM index $\left(\mathrm{g} / \mathrm{m}^{2}\right)$ & $102.8(21.6)$ & $83.4(16.6)$ & $<.001$ \\
\hline LV end-diastolic volume (mL) & $113.2(26.1)$ & $87.1(18.5)$ & $<.001$ \\
\hline LV end-diastolic volume index $\left(\mathrm{mL} / \mathrm{m}^{2}\right)$ & $55.6(11.5)$ & $49.3(9.5)$ & $<.001$ \\
\hline Interventricular septal thickness (cm) & $1.03(0.17)$ & $0.87(0.15)$ & $<.001$ \\
\hline Interventricular septal thickness index $\left(\mathrm{cm} / \mathrm{m}^{2}\right)$ & $0.50(0.08)$ & $0.49(0.08)$ & .006 \\
\hline Posterior wall thickness (cm) & $0.97(0.14)$ & $0.84(0.13)$ & $<.001$ \\
\hline Posterior wall thickness index $\left(\mathrm{cm} / \mathrm{m}^{2}\right)$ & $0.48(0.06)$ & $0.48(0.07)$ & .471 \\
\hline RWT & $0.37(0.06)$ & $0.35(0.06)$ & $<.001$ \\
\hline LA end-systolic volume (mL) & $60.5(18.0)$ & $50.5(14.8)$ & $<.001$ \\
\hline LA end-systolic volume index $\left(\mathrm{mL} / \mathrm{m}^{2}\right)$ & $29.6(8.1)$ & $28.5(7.4)$ & .021 \\
\hline LVEF Biplane (\%) & $60.3(5.9)$ & $61.7(5.7)$ & .001 \\
\hline Global strain $¥(\%)$ & $-19.5(2.4)$ & $-21.8(2.2)$ & $<.001$ \\
\hline$E / e^{\prime}$ & $7.0(1.5)$ & $7.5(1.7)$ & $<.001$ \\
\hline
\end{tabular}

The statistically significant associations between early growth and adult cardiac structure and adjusted models are presented in Table II. Some sex interactions in the associations between early growth and echocardiographic measures in midlife were observed, which are noted in the results.

Table 2. Associations between early growth and cardiac structure in midlife

\begin{tabular}{|c|c|c|c|c|c|c|c|c|c|c|c|c|}
\hline \multirow[t]{2}{*}{ Variables } & \multicolumn{2}{|c|}{ Model 1 (adjusted for sex) } & \multicolumn{3}{|c|}{ Model 2} & \multirow[b]{2}{*}{$P$ value } & \multicolumn{2}{|c|}{ Model 3} & \multirow[b]{2}{*}{$P$ value } & \multicolumn{2}{|c|}{ Model 4} & \multirow[b]{2}{*}{$P$ value } \\
\hline & $\mathrm{R}^{2}$ & $\beta(95 \% \mathrm{Cl})$ & $P$ value & $\mathrm{R}^{2}$ & $\beta(95 \% \mathrm{Cl})$ & & $\mathrm{R}^{2}$ & $\beta(95 \% \mathrm{Cl})$ & & $\mathrm{R}^{2}$ & $\beta(95 \% \mathrm{Cl})$ & \\
\hline \multicolumn{13}{|l|}{ LVM index } \\
\hline Age at $\mathrm{AR}$ male & 0.032 & $-4.10(-6.9,-1.3)$ & .004 & 0.071 & $-3.76(-6.6,-0.91)$ & .010 & 0.218 & $-3.30(-6.0,-0.56)$ & .018 & 0.258 & $-1.50(-4.4,1.4)$ & .305 \\
\hline Age at AR female & 0.003 & $-0.81(-2.5,0.93)$ & .361 & 0.012 & $-0.75(-2.6,1.1)$ & .424 & 0.097 & $-0.004(-1.8,1.8)$ & .997 & 0.136 & $1.22(-0.71,3.1)$ & .215 \\
\hline $\mathrm{BMI}$ at $\mathrm{AR}$ all & 0.203 & $2.33(0.80,3.9)$ & .003 & 0.214 & $2.28(0.62,3.9)$ & .007 & 0.300 & $1.86(0.23,3.5)$ & .026 & 0.331 & $0.61(-1.1,2.3)$ & .480 \\
\hline
\end{tabular}

LVEDV index 


\begin{tabular}{|c|c|c|c|c|c|c|c|c|c|c|c|c|}
\hline $\mathrm{BMI}$ at $\mathrm{AP}$ all & 0.100 & $1.47(0.51,2.4)$ & .003 & 0.112 & $1.21(0.17,2.3)$ & .023 & 0.237 & $1.35(0.31,2.4)$ & .011 & 0.238 & $1.34(0.30,2.4)$ & .012 \\
\hline Age at AR male & 0.038 & $-2.36(-3.9,-0.84)$ & .002 & 0.091 & $-2.45(-4.0,-0.91)$ & .002 & 0.290 & $-2.17(-3.7,-0.68)$ & .005 & 0.290 & $-2.13(-3.7,-0.51)$ & .010 \\
\hline Age at AR female & 0.001 & $-0.25(-1.3,0.79)$ & .639 & 0.028 & $-0.12(-1.2,0.95)$ & .820 & 0.116 & $-0.16(-1.2,0.91)$ & .772 & 0.117 & $-0.23(-1.4,0.91)$ & .690 \\
\hline $\mathrm{BMI}$ at $\mathrm{AR}$ all & 0.095 & $1.28(0.41,2.2)$ & .004 & 0.125 & $0.94(0.01,1.9)$ & .047 & 0.235 & $0.85(-0.07,1.8)$ & .070 & 0.235 & $0.82(-0.15,1.8)$ & .098 \\
\hline \multicolumn{13}{|l|}{ RWT } \\
\hline Birth weight all & 0.045 & $0.004(0.0,0.01)$ & .043 & 0.047 & $0.004(-0.001,0.009)$ & .095 & 0.148 & $0.006(0.001,0.01)$ & .016 & 0.159 & $0.005(0.0,0.011)$ & .046 \\
\hline \multicolumn{13}{|l|}{ LAESV index } \\
\hline Age at AR male & 0.038 & $-1.55(-2.5,-0.55)$ & .002 & 0.061 & $-1.56(-2.6,-0.53)$ & .003 & 0.228 & $-1.57(-2.6,-0.52)$ & .004 & 0.272 & $-0.84(-1.9,0.27)$ & .137 \\
\hline Age at AR female & 0.015 & $-0.89(-1.7,-0.09)$ & .028 & 0.022 & $-0.96(-1.8,-0.13)$ & .023 & 0.076 & $-0.93(-1.8,-0.09)$ & .030 & 0.095 & $-0.56(-1.4,0.32)$ & .212 \\
\hline $\mathrm{BMI}$ at $\mathrm{AR}$ male & 0.019 & $1.12(0.08,2.2)$ & .036 & 0.032 & $0.87(-0.29,2.0)$ & .143 & 0.201 & $0.96(-0.27,2.2)$ & .126 & 0.265 & $0.14(-1.1,1.4)$ & .830 \\
\hline $\mathrm{BMI}$ at $\mathrm{AR}$ female & 0.005 & $0.49(-0.27,1.2)$ & .206 & 0.011 & $0.57(-0.24,1.4)$ & .168 & 0.063 & $0.40(-0.44,1.2)$ & .349 & 0.090 & $0.03(-0.84,0.89)$ & .955 \\
\hline
\end{tabular}

$A P$, adiposity peak; $A R$, adiposity rebound; $L A E S V$, left atrial end-systolic volume; $L V E D V$, left ventricular end-diastolic volume. All significant associations between early growth and adult cardiac structure in model 1 are shown. Sexes were analyzed together except for when an interaction by sex in associations was observed. When a significant association was observed in either sex, results for both sexes are presented. The values are statistical significances from linear regression models $(P)$, explained variance of the model $\left(R^{2}\right)$, and unstandardized beta $(\beta)$ with $95 \% \mathrm{Cl}$ for $1 \mathrm{SD}$ increase in main independent variable.

Echocardiographic measures are indexed to body surface area, except for RWT. Model 1: adjustment for sex; Model 2: model 1 and birth weight, maternal prepregnancy BMI, and family SES (and gestational age only for analysis with birth weight as main independent variable); Model 3 : Model 2 and lifelong physical activity as well as adult heart rate, systolic and diastolic blood pressure, diabetes, cardiac diseases, and antihypertensive medication; Model 4: Model 3 and adult BMI (+height for nonindexed RWT). $n$ = 782-1014 for birth weight, 400-482 at adiposity peak, 488-586 at adiposity rebound.

Gestational age was not significantly associated with adult cardiac structure (data not shown). Higher birth weight was related to increased RWT, even after all relevant adjustments (Table II).

Age at adiposity peak was not associated with cardiac structure in adulthood. Associations between timing of adiposity rebound and later cardiac structure were partly sex specific. In male participants, earlier adiposity rebound was related to increased LVM index and LV end-diastolic volume index. The relationship between timing of adiposity rebound and LVM index remained significant after adjustment for birth and maternal variables, lifelong physical activity, and adult cardiovascular risk factors and morbidity, but not when further adjusted for adult BMI. The association between earlier adiposity rebound and higher LV end-diastolic volume index remained significant after all relevant adjustments. The association between earlier adiposity rebound and higher LV end-diastolic volume index was also independent of BMI at adiposity peak $\left(\mathrm{R}^{2}=0.333 \beta=-2.41[-4.0,-0.79], \mathrm{P}=.004\right)$. In female participants, similar findings were not found. Age at adiposity rebound was indirectly associated with LA end-systolic volume index in both sexes, independent of maternal and birth variables, lifelong physical activity, and adult cardiovascular risk factors and morbidity (Table II).

BMI at adiposity rebound remained directly associated with LVM index after adjustment for all the other relevant factors except adult BMI. Higher BMI at 
adiposity peak and at adiposity rebound were both related to increased LV enddiastolic volume index. The association between higher BMI at adiposity peak and LV end-diastolic volume index remained significant after all relevant adjustments including adjustment for adult BMI. BMI at adiposity rebound had a direct association with a higher LA end-systolic volume index in males in the model adjusted for sex (Table II).

Birth weight was indirectly associated with $\mathrm{E} / \mathrm{e}$ ' in the model adjusted for sex $\left(\mathrm{R}^{2}=0.031, \beta=-0.011[-0.21,-0.002], \mathrm{P}=.045\right)$ and after adjustment for birth and maternal variables $\left(\mathrm{R}^{2}=0.034, \beta=-0.18[-0.31,-0.05], \mathrm{P}=.005\right)$. However, the association was attenuated after adjustment for lifelong physical activity and adult cardiovascular risk factors and morbidity, and further adjustment for adult anthropometrics (BMI and height) $\left(\mathrm{R}^{2}=0.138, \beta=-0.13[-0.27,0.005], \mathrm{P}=.060\right.$; $\mathrm{R}^{2}=0.169, \beta=-0.10$ [-0.24, 0.04], $\mathrm{P}=.168$, respectively). No other significant associations were observed between early growth variables and E/e', LVEF, or global strain.

Gestational age, birth weight, and age at adiposity peak did not differ significantly in LV remodeling categories. Eccentric hypertrophy was associated with a higher BMI at adiposity peak and at adiposity rebound even after adjustment for birth and maternal variables, lifelong physical activity, adult cardiovascular risk factors and morbidity, and adult BMI. But these associations did not remain significant if BMI at adiposity peak and at adiposity rebound were included in the model at the same time (OR $1.31[0.89,1.92], \mathrm{P}=.178$ for BMI at adiposity peak and OR $1.26[0.85,1.86], \mathrm{P}=.259$ for BMI at adiposity rebound). Concentric remodeling in adulthood was related to higher BMI at adiposity peak and at adiposity rebound in sex-adjusted models. There were sex differences in the relationships between age at adiposity rebound and LV remodeling categories. Eccentric hypertrophy was associated with earlier adiposity rebound even in the final multivariate model in males. Further adjustment of the association between eccentric hypertrophy and age at adiposity rebound in male participants for BMI at adiposity peak did not attenuate the association (OR 0.45 [0.26, 0.77], $\mathrm{P}=.004)$. Concentric remodeling had a significant association with earlier adiposity rebound in female participants in the sex-adjusted model (Table III).

Table 3. Associations between early growth and left ventricular remodeling categories in midlife 
OR $(95 \% \mathrm{Cl})$

Model 1 (adjusted for sex)

Gestational age all

Birth weight all

Age at adiposity peak all

$\mathrm{BMI}$ at adiposity peak all

Age at adiposity rebound male

Age at adiposity rebound female

$\mathrm{BMI}$ at adiposity rebound all

\section{Model 2}

BMI at adiposity peak all

Age at adiposity rebound male

Age at adiposity rebound female

$\mathrm{BMI}$ at adiposity rebound all

$\begin{array}{ll}1.01(0.85,1.21) & .891 \\ 1.17(0.99,1.40) & .075 \\ 1.04(0.81,1.34) & .743 \\ 1.41(1.10,1.82) & .007 \\ 0.54(0.38,0.77) & .001 \\ 0.84(0.63,1.13) & .261 \\ 1.53(1.23,1.91) & <.001\end{array}$

$0.99(0.80,1.23)$

$1.22(0.99,1.50)$

$1.09(0.81,1.48)$

$1.38(1.02,1.87)$

$0.97(0.65,1.45)$

$0.68(0.46,0.99)$

$1.40(1.06,1.83)$

.008
.001
.463
.001

$1.28(0.92,1.78)$

.142

.736

$0.93(0.61,1.42)$

$0.73(0.48,1.11)$

.138

$1.27(0.94,1.71)$

.118

$\begin{array}{ll}0.89(0.66,1.21) & .463 \\ 1.50(1.19,1.90) & .001\end{array}$

.008

$1.28(0.88,1.87)$

.191

.410

.320

.148
OR $(95 \% \mathrm{Cl})$

$0.88(0.69,1.13) \quad .882$

$1.10(0.85,1.42) \quad .487$

$0.82(0.56,1.19) \quad .296$

$1.12(0.77,1.63) \quad .556$

$0.82(0.50,1.37) \quad .451$

$1.02(0.62,1.68) \quad .951$

$1.23(0.87,1.75) \quad .242$

$\begin{array}{ll}1.02(0.68,1.53) & .914 \\ 0.80(0.47,1.34) & .396 \\ 1.03(0.61,1.72) & .915 \\ 1.16(0.80,1.68) & .431\end{array}$

$0.92(0.56,1.49) \quad .727$

$0.70(0.38,1.29) \quad .254$

$1.17(0.65,2.14) \quad .598$

$1.07(0.69,1.65) \quad .757$

\section{Model 4}

BMI at adiposity peak all

Age at adiposity rebound male

Age at adiposity rebound female

$\mathrm{BMI}$ at adiposity rebound all

All associations between early growth and adult

$1.47(1.09,1.98) \quad .012$

$0.51(0.32,0.80)$

.004

.665

$1.41(1.08,1.83) \quad .012$ association was observed in either sex in model 1, further adjusted results for both sexes are presented. The values are statistical significances for multinomial logistic regression models $(P)$ and OR with $95 \% \mathrm{CI}$ for left ventricular remodeling per $1 \mathrm{SD}$ increase in independent variable. Model 1: adjustment for sex; Model 2:

Model 1 and birth weight, maternal pre-pregnancy BMI, and family SES; Model 3: Model 2 and lifelong physical activity as well as adult heart rate, systolic and diastolic blood pressure, diabetes, cardiac diseases, and antihypertensive medication; Model 4: Model 3 and adult BMI. $n=977-1010$ for birth weight and gestational age, 413480 at adiposity peak, 502-584 at adiposity rebound. 


\section{Discussion}

Timing of adiposity rebound involves the examination of several points in growth, giving further insight into BMI patterns leading to future overweight and cardiometabolic risk. Early adiposity rebound has been shown to reflect greater weight gain, particularly accumulation of body fat rather than lean mass, in childhood (32,33). Although lean mass is the most important determinant of childhood cardiac growth, increased adiposity has been shown to be a major factor related to excess cardiac growth and cardiac remodeling (14-16,34). This is in line with our finding of associations between earlier adiposity rebound and greater LVM, LV size, and eccentric hypertrophy in adult males. Earlier adiposity rebound was also related to greater adult LA size in both sexes. LA size describes the influence of diastolic LV filling pressure over time. In addition to reflecting chronic diastolic dysfunction and its severity, increased LA size has been associated with an augmented risk of cardiovascular events and atrial fibrillation (35).

Obesity and LVM have been shown to track from childhood to later life and predict cardiovascular risk and morbidity $(17,18,36,37)$. Early childhood has been suggested to be a critical period for development of sustained obesity (36). Higher BMI as early as at adiposity peak in infancy has been related with later overweight $(6,24)$. We found that BMI at infant adiposity peak (at average age 9 months) and at childhood adiposity rebound (at average age 5.8 years) were both associated with adult cardiac structure. These findings were partly mediated by adult BMI, which is in line with previous studies suggesting that the cumulative burden of obesity beginning in childhood and persisting into adulthood has a deleterious effect on future cardiac structure $(9,19-22)$. Adaptations in LV structure caused by obesity have been observed in children at as early as at 2 years of age (16), and these may persist into later life, although the confirmation of this concept requires further studies. However, in our study, associations of higher BMI at adiposity peak with greater adult LV end-diastolic volume as well as associations of higher BMI at adiposity peak and at adiposity rebound with adult eccentric hypertrophy were not fully explained by adult BMI suggesting that early growth patterns may influence adult cardiac structure also through other mechanisms than through the indirect effect of the tracking of BMI.

Obesity may influence myocardial growth through various interrelated pathways, including hemodynamic, neurohormonal, metabolic, and genetic mechanisms, leading to increase in LV size, hypertrophy, and remodeling (38). Overweight and obesity are related to a chronic volume overload and an elevated 
cardiac output, and excessive adiposity has been shown to be closely related to eccentric hypertrophy (20). Obesity is also related to sympathetic over activity and adipose tissue may cause renin-angiotensin induced myocardial growth and fibrosis (39). Genetic predisposition and epigenetic mechanisms may also be involved $(38,40)$. However, the mechanisms behind our present observations are beyond the scope of this study and merit further studies.

In our study, infant and childhood BMI growth patterns contributed more than fetal growth to adult cardiac structure. We found that higher birth weight was associated with increased adult cardiac RWT. Previous studies have also observed birth weight to be associated with LV wall thickness in adolescents (41), but similar findings have not been observed in adulthood $(9,11,13)$. We did not find an association between birth weight and adult LVM or LV size when body size and sex were taken into account, findings which are in alignment with previous observations (9,11-13). In our study, birth weight tended to associate with LV concentric remodeling and the associations that we observed between childhood growth and concentric remodeling were not independent of birth weight and maternal factors. This suggests that prenatal influences may partly contribute to later cardiac structure indirectly through childhood growth patterns. In our study, lower birth weight tended to associate with larger E/e'. Similar results have been reported in children with fetal growth restriction and in those born small for gestational age $(42,43)$. Premature birth has previously been related to adverse cardiac adaptations in adulthood (8), however, in our study gestational age was not associated with adult cardiac structure. This may be because early preterm survival rate in the year 1966 was lower, which may have reduced the number of premature live births in our study sample.

There are some limitations to our study. The study sample did not fully represent the whole NFBC 1966. All observed effect estimates were relatively small, and the clinical importance of the minor early growth-related changes observed in adult cardiac structure can be debated. However, the observed associations should be considered from the perspective of life course trajectories. It is also noteworthy that we were not able to adjust the relationships between early growth and adult cardiac structure for all potential confounders. Finally, as obesity tracks strongly from childhood to adulthood, it is challenging to detach the influence of early growth from current body size.

In conclusion, early BMI growth patterns, specifically earlier adiposity rebound as well as higher BMI at adiposity peak and at adiposity rebound, contributed to modifications in cardiac structure at midlife. Birth weight was a less 
important determinant of adult cardiac structure in our study. LV function in adulthood was not related to any of the early growth variables after relevant adjustments.

We thank the late professor Paula Rantakallio (launch of NFBC 1966), the participants in the 46-year study, and the NFBC project center (cohort administration).

\section{References}

1. D. Levy, R.J. Garrison, D.D. Savage, W.B. Kannel and W.P. Castelli, Prognostic implications of echocardiographically determined left ventricular mass in the Framingham Heart Study, N Engl J Med 322, 1990, 1561-1566.

2. D. Levy, R.J. Garrison, D.D. Savage, W.B. Kannel and W.P. Castelli, Left ventricular mass and incidence of coronary heart disease in an elderly cohort: the Framingham Heart Study, Ann Intern Med 110, 1989, 101-107.

3. J.M. Gardin, R. McClelland, D. Kitzman, J.A. Lima, W. Bommer, H.S. Klopfenstein, et al., M-mode echocardiographic predictors of six- to seven-year incidence of coronary heart disease, stroke, congestive heart failure, and mortality in an elderly cohort (the Cardiovascular Health Study), Am J Cardiol 87, 2001, 1051-1057.

4. D.J. Barker, C. Osmond, J. Golding, D. Kuh and M.E. Wadsworth, Growth in utero, blood pressure in childhood and adult life, and mortality from cardiovascular disease, BMJ 298, 1989, 564-567.

5. S. Peneau, R. Gonzalez-Carrascosa, G. Gusto, D. Goxe, O. Lantieri, L. Fezeu, et al., Age at adiposity rebound: determinants and association with nutritional status and the metabolic syndrome at adulthood, Int J Obes 40, 2016, 1150-1156.

6. U. Sovio, M. Kaakinen, I. Tzoulaki, S. Das, A. Ruokonen, A. Pouta, et al., How do changes in body mass index in infancy and childhood associate with cardiometabolic profile in adulthood? Findings from the Northern Finland Birth Cohort 1966 Study, Int J Obes 38, 2014, 53-59.

7. L.J. Lloyd, S.C. Langley-Evans and S. McMullen, Childhood obesity and adult cardiovascular disease risk: a systematic review, Int J Obes 34, 2010, 18-28.

8. A.J. Lewandowski, D. Augustine, P. Lamata, E.F. Davis, M. Lazdam, J. Francis, et al., Preterm heart in adult life: cardiovascular magnetic resonance reveals distinct differences in left ventricular mass, geometry, and function, Circulation 127, 2013, 197-206.

9. R. Hardy, A.K. Ghosh, J. Deanfield, D. Kuh and A.D. Hughes, Birthweight, childhood growth and left ventricular structure at age 60-64 years in a British birth cohort study, Int $J$ Epidemiol 45, 2016, 1091-1102. 
10. C. Arnott, M.R. Skilton, S. Ruohonen, M. Juonala, J.S. Viikari, M. Kahonen, et al., Subtle increases in heart size persist into adulthood in growth restricted babies: The Cardiovascular Risk in Young Finns Study, Open Heart 2, 2015, e000265.

11. M. Vijayakumar, C.H. Fall, C. Osmond and D.J. Barker, Birthweight, weight at one year, and left ventricular mass in adult life, Br Heart J 73, 1995, 363-367.

12. N. Bjarnegård, E. Morsing, M. Cinthio, T. Länne and J. Brodszki, Cardiovascular function in adulthood following intrauterine growth restriction with abnormal fetal blood flow, Ultrasound Obstet Gynecol 41, 2013, 177-184.

13. M. Zureik, C. Bonithon-Kopp, E. Lecomte, G. Siest and P. Ducimetirere, Weights at birth and in early infancy, systolic pressure, and left ventricular structure in subjects aged 8 to 24 years, Hypertension 27, 1996, 339-345.

14. S. Dhuper, R.A. Abdullah, L. Weichbrod, E. Mahdi and H.W. Cohen, Association of obesity and hypertension with left ventricular geometry and function in children and adolescents, Obesity (Silver Spring) 19, 2011, 128-133.

15. E.M. Urbina, S.S. Gidding, W. Bao, A.S. Pickoff, K. Berdusis and G.S. Berenson, Effect of body size, ponderosity, and blood pressure on left ventricular growth in children and young adults in the Bogalusa Heart Study, Circulation 91, 1995, 2400-2406.

16. L.L. de Jonge, L. van Osch-Gevers, S.P. Willemsen, E.A. Steegers, A. Hofman, W.A. Helbing, et al., Growth, obesity, and cardiac structure in early childhood: the Generation R Study, Hypertension 57, 2011, 934-940.

17. R.M. Schieken, P.F. Schwartz and M.M. Goble, Tracking of left ventricular mass in children: race and sex comparisons - the MCV Twin Study: Medical College of Virginia, Circulation 97, 1998, 1901-1906.

18. S. Sivanandam, A.R. Sinaiko, D.R. Jacobs, L.M. Steffen, A. Moran and J. Steinberger, Relation of increase in adiposity to increase in left ventricular mass from childhood to young adulthood, Am J Cardiol 98, 2006, 411-415.

19. X. Li, S. Li, E. Ulusoy, W. Chen, S.R. Srinivasan and G.S. Berenson, Childhood adiposity as a predictor of cardiac mass in adulthood: the Bogalusa Heart Study, Circulation 110, 2004, 3488-3492.

20. A. Toprak, H. Wang, W. Chen, T. Paul, S. Srinivasan and G. Berenson, Relation of childhood risk factors to left ventricular hypertrophy (eccentric or concentric) in relatively young adulthood (from the Bogalusa Heart Study), Am J Cardiol 101, 2008, 1621-1625.

21. C.-C. Lai, D. Sun, R. Cen, J. Wang, S. Li, C. Fernandez-Alonso, et al., Impact of longterm burden of excessive adiposity and elevated blood pressure from childhood on adulthood left ventricular remodeling patterns. The Bogalusa Heart Study, J Am Coll Cardiol 64, 2014, 1580-1587. 
22. H. Zhang, T. Zhang, S. Li, Y. Guo, W. Shen, C. Fernandez, et al., Long-term excessive body weight and adult left ventricular hypertrophy are linked through later body size and blood pressure: the Bogalusa Heart Study, Circ Res 120, 2017, 1614-1621.

23. M.F. Rolland-Cachera, M. Deheeger, M. Maillot and F. Bellisle, Early adiposity rebound: causes and consequences for obesity in children and adults, Int J Obes 30, 2006, 11-17.

24. R.J. Silverwood, B.L. De Stavola, T.J. Cole and D.A. Leon, BMI peak in infancy as a predictor for later BMI in the Uppsala Family Study, Int J Obes 33, 2009, 929-937.

25. M.R. Järvelin, U. Sovio, V. King, L. Lauren, B. Xu, M.I. McCarthy, et al., Early life factors and blood pressure at age 31 years in the 1966 Northern Finland birth cohort, Hypertension 44, 2004, 838-846.

26. P. Oura, M. Paananen, J. Niinimäki, T. Tammelin, S. Herrala, J. Auvinen, et al., Effects of leisure-time physical activity on vertebral dimensions in the Northern Finland Birth Cohort 1966, Sci Rep 6, 2016, 27844.

27. A.M. Kiviniemi, N. Perkiömäki, J. Auvinen, S. Herrala, A.J. Hautala, R. Ahola, et al., Lifelong physical activity and cardiovascular autonomic function in midlife, Med Sci Sports Exerc 48, 2016, 1506-1513.

28. K.G. Alberti and P.Z. Zimmet, Definition, diagnosis and classification of diabetes mellitus and its complications. Part 1: diagnosis and classification of diabetes mellitus provisional report of a WHO consultation, Diabet Med 15, 1998, 539-553.

29. R.M. Lang, M. Bierig, R.B. Devereux, F.A. Flachskampf, E. Foster, P.A. Pellikka, et al., Task Force on Chamber Quantification; American College of Cardiology Echocardiography Committee; American Heart Association; European Association of Echocardiography, European Society of Cardiology. Recommendations for chamber quantification: a report from the American Society of Echocardiography's Guidelines and Standards Committee and the Chamber Quantification Writing Group, developed in conjunction with the European Association of Echocardiography, a branch of the European Society of Cardiology, J Am Soc Echocardiogr 18, 2005, 1440-1463.

30. A.P. Patrianakos, A.A. Zacharaki, A. Kalogerakis, G. Solidakis, F.I. Parthenakis and P.E. Vardas, Two-dimensional global and segmental longitudinal strain: are the results from software in different high- end ultrasound systems comparable?, Echo Res Pract 2, 2015, 29-39.

31. O.A. Smiseth, H. Torp, A. Opdahl, K.H. Haugaa and S. Urheim, Myocardial strain imaging: how useful is it in clinical decision making?, Eur Heart J 37, 2015, 1196-1207.

32. R.W. Taylor, A. Goulding, N.J. Lewis-Barned and S.M. Williams, Rate of fat gain is faster in girls undergoing early adiposity rebound, Obes Res 12, 2004, 1228-1230.

33. S.M. Williams, Weight and height growth rate and the timing of adiposity rebound, Obes

Res 13, 2005, 1123-1130. 
34. S.R. Daniels, T.R. Kimball, J.A. Morrison, P. Khoury, S. Witt and R.A. Meyer, Effect of lean body mass, fat mass, blood pressure, and sexual maturation on left ventricular mass in children and adolescents: statistical, biological, and clinical significance, Circulation 92, 1995, 3249-3254.

35. T.S. Tsang, M.E. Barnes, B.J. Gersh, K.R. Bailey and J.B. Seward, Left atrial volume as a morphophysiologic expression of left ventricular diastolic dysfunction and relation to cardiovascular risk burden, Am J Cardiol 90, 2002, 1284-1289.

36. M. Geserick, M. Vogel, R. Gausche, T. Lipek, U. Spielau, E. Keller, et al., Acceleration of BMI in early childhood and risk of sustained obesity, N Engl J Med 379, 2018, 13031312.

37. L. Toemen, R. Gaillard, L. van Osch-Gevers, W.A. Helbing, A. Hofman and V.W. Jaddoe, Tracking of structural and functional cardiac measures from infancy to school-age, Eur J Prev Cardiol 24, 2017, 1408-1415.

38. E.D. Abel, S.E. Litwin and G. Sweeney, Cardiac remodeling in obesity, Physiol Rev 88, 2008, 389-419.

39. S. Engeli, R. Negrel and A.M. Sharma, Physiology and pathophysiology of the adipose tissue renin-angiotensin system, Hypertension 35, 2000, 1270-1277.

40. L. Benschop, S. Schalekamp-Timmermans, J.E. Roeters van Lennep, V.W.V. Jaddoe, E.A.P. Steegers and M.K. Ikram, Cardiovascular risk factors track from mother to child, $J$ Am Heart Assoc 7, 2018, e009536.

41. H. Hietalampi, K. Pahkala, E. Jokinen, T. Ronnemaa, J.S. Viikari, H. Niinikoski, et al., Left ventricular mass and geometry in adolescence: early childhood determinants, Hypertension 60, 2012, 1266-1272.

42. F. Crispi, B. Bijnens, F. Figueras, J. Bartrons, E. Eixarch, F. Le Noble, et al., Fetal growth restriction results in remodeled and less efficient hearts in children, Circulation 121, 2010, 2427-2436.

43. S. Timpka, A.D. Hughes, N. Chaturvedi, P.W. Franks, D.A. Lawlor, J.W. Rich-Edwards, et al., Birth weight and cardiac function assessed by echocardiography in adolescence: Avon Longitudinal Study of Parents and Children, Ultrasound Obstet Gynecol 54, 2019, 225-231 